\title{
Evaluation of Multipoint Contact Interfaces in Haptic Perception of Shapes
}

\author{
Antonio Frisoli ${ }^{1}$, Massimo Bergamasco ${ }^{1}$, Sue-Lynn $\mathrm{Wu}^{1}$, and Emanuele Ruffaldi ${ }^{1}$ \\ PERCRO Scuola Superiore Sant'Anna, Pisa, Italy, a.frisoli@ sssup.it \\ http://www.percro.org
}

\begin{abstract}
This paper presents the evaluation of a multicontact point interface for the shape recognition of objects. A set-up constituted of an Haptic Interface system with several contact points, from one up to three, was used to present to four different subjects a set of 6 basic shapes. The experimental results suggest that the number of contact points do not produce an improvement of the ability to identify shapes, and this is strongly in contrast with what happens in touch with bare fingers. These findings confirmed results already found in other works, where experiments were conducted with fingers covered by sheaths, and provide indications for the design of more effective multipoint haptic interfaces.
\end{abstract}

\section{Introduction}

Haptic interaction with virtual environments is ideally meant to provide an intuitive method of exploration. However, the reduction of interaction points from the natural ten fingers to one contact point, as is often the case haptics, hinders haptic perception (8). (12) have shown that haptic perception by free exploration with hands can be accurate and fast. This type of performance, however, has not been duplicated in experiments with constrains on the number and natural movement of fingers. (10) found that free hand exploration is more efficient than outstretched five-finger exploration, which was subsequently more efficient than one outstretched finger. (6) discovered a significant detriment in performance when only one finger was available for exploration of common objects. In an earlier experiment, also reported in (8), he found that numbers of fingers had a great effect on the efficiency in identification of real objects, the largest difference appearing between the One finger and the Two fingers conditions. However, the Two fingers conditions were represented by only one such combination, thumb and index finger.

A further experiment conducted by the same author involved an haptic display condition with not differentiated information within the contact region, simulated by a hard sheath attached to the finger(s) in contact with the objects, similar to the one used by (11) when studying some other haptic functions.

A personal communication from the same author, supported by experimental data reported in an internal report (7), confirmed that in the Sheath conditions there was no difference in Exploration time at all between the One finger and the Two fingers conditions, according to the experimental findings.

This would suggest that the effect of restrictions on the fingerpad contact region can limit the added value of having multiple contact points on more fingers. Actually 
this is the case of Haptic Interfaces based on kinesthetic feedback, that do not provide at all information about the contact location.

The aim of the present experiment was to compare the efficiency of some of the possible two fingers combinations, when using kinesthetic haptic interfaces enabling multipoint interaction.

\section{Motivation}

It should be noted that more than one point of is not always a requirement. As both (9) and (1) stated in past works, the haptic mode is dependent on the application at hand. For example, tasks such as determining temperature, and hardness only require one point of contact. Tasks such as determining shape and size of an object are more efficient with more than one point of contact.

When interacting with the environment (whether real or virtual) human's come into contact with many objects. The first action is to determine what type of object is presented. This takes the form of exploring the general shape of the object. Only after establishing the object's general shape and size, does the investigator attend to less critical characteristics such as specific object contours. The first stage of investigation is dominated by actions such as enclosure (envelopment of the object with the hand) and unsupported holding (lifting of object above supporting surface). These actions require more than one finger. Without the possibility to interact in this manner, the investigator resorts to contour following (tracing edges) to resolve the shape and part characteristics of the object. This procedure, however, is much less efficient (9).

Blind individuals rely strongly on haptic interaction with the world. Often, when information is not sufficient through investigations with one hand, such as when trying to process the face of a new acquaintance, the blind individual will enhance information collection through the addition of the other hand into the investigation. (3) has shown that processing of vibrotactile patterns is more efficient when the patterns are presented to two fingers on opposite hands than on the same hand. The physical distinction and separation of the two hands appears to make information processing easier. (4) also found that separation between fingers in exploration with one hand enhances perception of curvature and permits a global perception of the object. The physical separation helped the participant to"organize successive parts of a form into a whole, thereby reducing the requirement for integrating successive inputs in memory".

In the study described in this paper, we explore the difference in performance for object recognition as the number of contact points is increased using either the right hand or both. We focus on geometric perception because it is fundamental to interaction with the environment. We include observation with two hands to closer mimic real-world interaction with the environment. Findings from this study can be used to improve performance in such environments as haptic training systems, and information presentation to the blind. 


\section{Methods}

\subsection{Subjects}

Four people, selected among students and employees at the Scientific and Technology Centre of the Scuola Superiore Sant'Anna, participated in the study without monetary compensation.

\subsection{Haptic Interfaces}

Haptic interfaces available at the Centre were used to investigate interactions with the following combination of contact points:

1. Index finger on right hand

2. Thumb and index finger on right hand

3. Thumb, index and middle finger on right hand.

Right hand interactions are primary in this study because it is assumed that a great majority of the participants are right-handed.

The 3 contact points condition was achieved by adopting two haptic interface system enabling two contact point with the users.

One device, the PURE-FORM hand exoskeleton device, is described in (5) and shown in Figure 1. It was designed to be used in combination with an arm exoskeleton device, considered worn by the operator on his arm as shown in Figure 2 , providing the force feedback on index and thumb fingers of the same hand. It is characterized by very low friction and inertias, being the actuators located on the grounded link, and by good dynamic performance. In reason of its large workspace, in the present experiment, it was mounted in the configuration shown in Figure 1, facing the operator's hand.

In order to provide up to 4 contact points to the operator, the hand-exoskeleton was used in combination with another 2 contact points device, the GRAB system, specifically developed by PERCRO in a previous EU project as a technlogical aid for blind people.

This device is characterized by a larger workspace, and can be used with two fingers of the same or different hand. On the other side, in reason of its larger workspace, the GRAB device is less transparent than the hand-exoskeleton, so that when both ones are used in combination, there is a difference of perceived inertia among the fingers.

\subsection{Shapes}

Two common shapes are used for the training phase of the investigations.

1. Sphere: $10 \mathrm{~cm}$ diameter

2. Cube: $10 \mathrm{~cm}$ side

Basic shapes are used for the experiments. These shapes are as follows: 


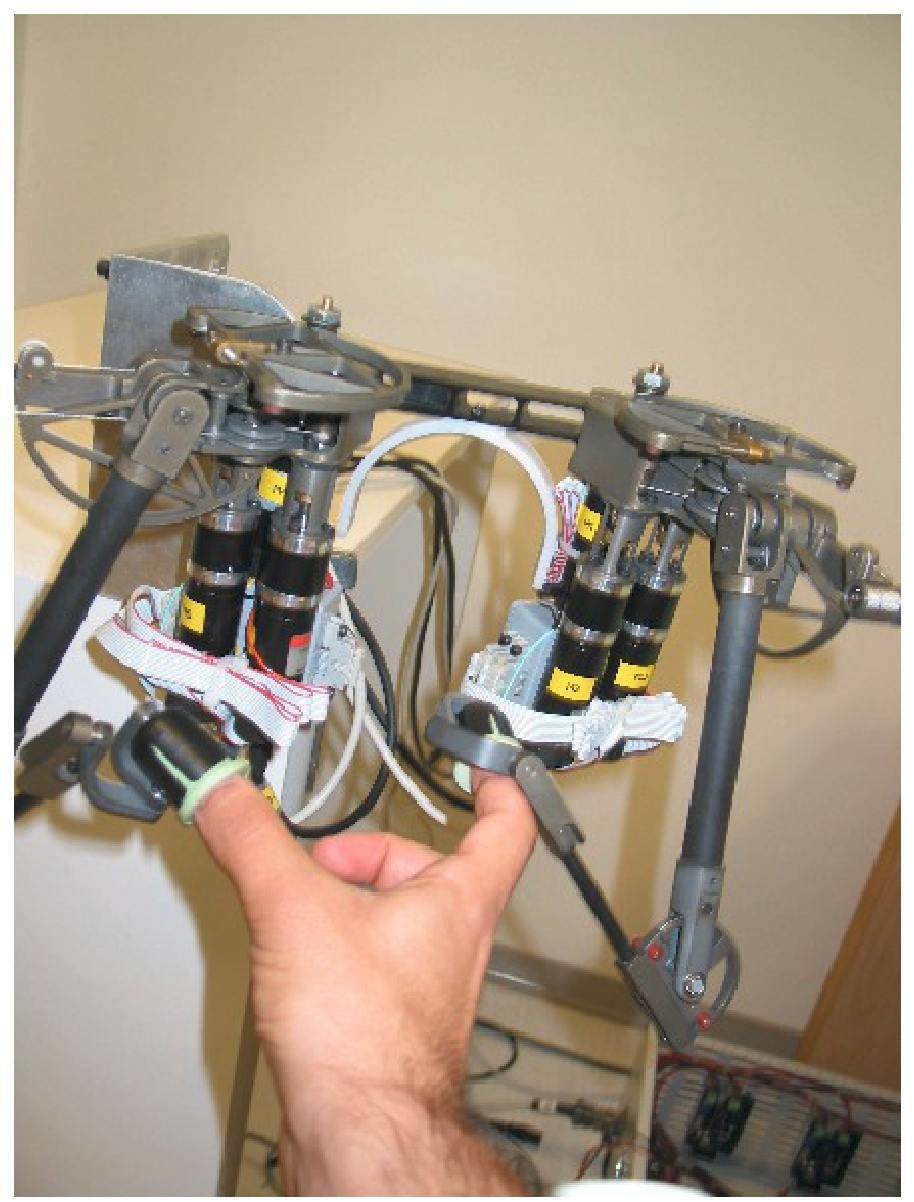

Fig. 1. Set-up of the haptic system with two available contact points

1. Cylinder: $10 \mathrm{~cm}$ diameter; $10 \mathrm{~cm}$ height

2. Cone: $10 \mathrm{~cm}$ diameter; $10 \mathrm{~cm}$ height

3. Pyramid: square base with $10 \mathrm{~cm}$ sides; $10 \mathrm{~cm}$ height

4. Half-sphere with flat facing upward: $10 \mathrm{~cm}$ diameter

5. Triangular extrusion: $10 \mathrm{~cm}$ base; $10 \mathrm{~cm}$ height; $10 \mathrm{~cm}$ length

6. Pentagon extrusion: circumvented by circle of $10 \mathrm{~cm}$ diameter

Basic shapes were chosen because (6) found that participants attempting to recognize very basic shapes in the virtual environment (VE), with one point of contact, could not reach a performance level of $100 \%$ with respect to natural exploration with real objects; performance was $80 \%$ at best. In investigations that advanced beyond basic shapes, Jansson found an increase in exploration time and decrease in correct responses as complexity increased. 


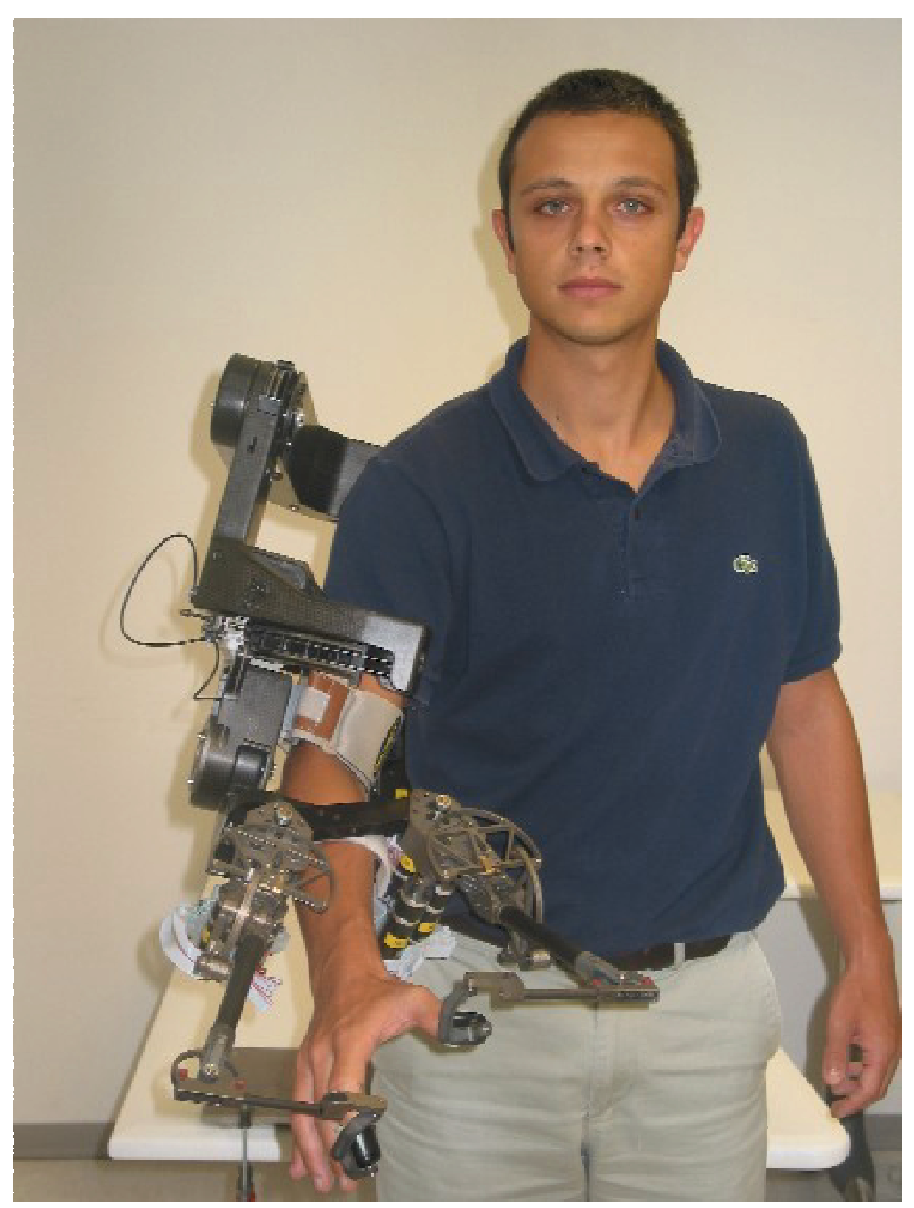

Fig. 2. The arm exoskeleton with hand exoskeleton system

In addition, (1) found error rates of $15 \%$ with VE representations of simple smooth curves. Further, (2) found that participants had difficulty reconstructing complex shapes. Thus, recognition of objects that are more complicated than basic shapes should not be attempted until further knowledge is gained from experiments with basic shapes.

The size of the shapes was chosen based on Kirkpatrick's and Jansson's findings that larger shapes are correlated to shorter performance times. In Kirkpatrick's experiments, the stimuli spanned up to $7 \mathrm{~cm}$. In (author?)'s experiments, the objects were characterized by dimensions up to $10 \mathrm{~cm}$. Consequently, we chose the larger of the two figures for the present study. 


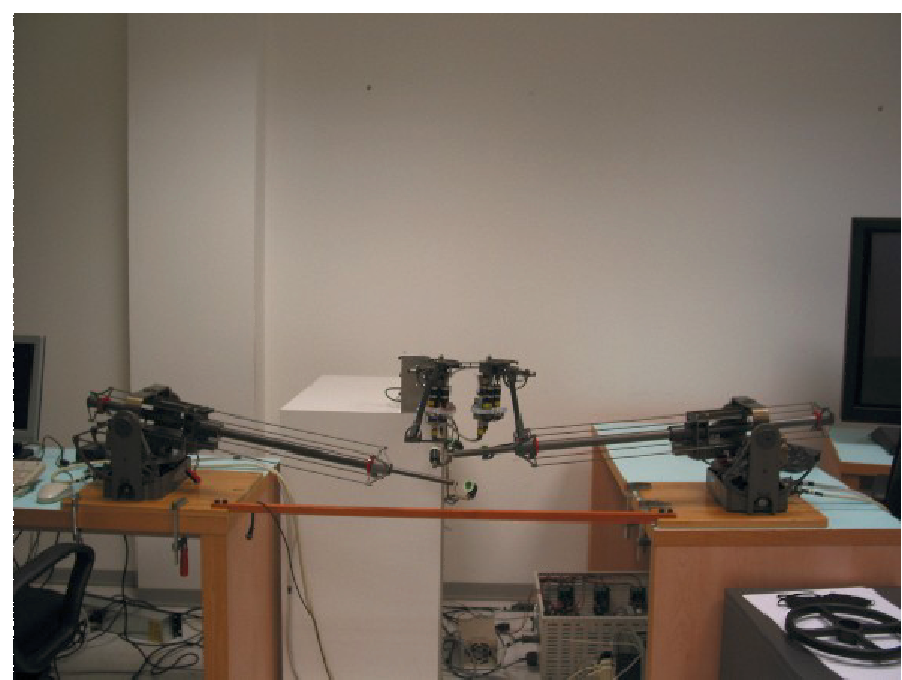

Fig. 3. Set-up of the haptic system with four available contact points

\subsection{Training Phase}

Because studies by (6) have shown that displays with users without practice underestimate their usefulness, participants in this study are given training with each of the haptic interfaces prior to the actual experiment. Participants are allowed to feel the sphere and cube previously described. Practice continues until the participant can identify the shape with each interface using the maximum number of contact points available on the particular interface.

The purpose of the practice phase is to enable the participant to become familiar with the haptic interfaces and the sensation of "feeling the invisible objects". Each of the three contact-point combinations are introduced to the participant at this point.

1. Two different haptic interfaces are used for these experiments. Figure 3 shows the interface from PERCRO.

2. Participant stand with the haptic interface positioned at forearm length directly in front of the torso.

3. The practice session begins first with practice with visual feedback and then without.

4. The contact points are introduced to the participant in increasing order. Using the participant's dominant hand, the appropriate finger(s) are placed into the thimble of the interface according to the contact points under examination. PERCRO Setup: i. One Point: Interface One with index finger ii. Two Points: Interface One with index and thumb

5. A dragging force directs the finger(s) to the object at the beginning of each shape introduction.

6. A cube $(10 \mathrm{~cm}$ diameter $)$ is introduced to the participants in both the one and two contact point phases. 
7. Often during exploration, participants may experience lost of contact with the object. To limit the amount of time spent in open space, a spherical bubble $(9 \mathrm{~cm}$ radius with the same origin as the object) encloses the object. Each time the participant remains outside this sphere for more than three seconds, the dragging force guides the finger(s) back to the shape.

8. At the beginning of the practice phase, the participant reads a set of instructions that state the following:

"Practice until you become comfortable with the interface.

If you stay outside the field of the object, a force will drag your finger(s) to the shape. I will help you position your blindfold over your eyes.

Please begin your exploration when I say GO".

9. A blindfold is put over the participant's eyes to both block vision of the hand(s) and to eliminate any visual inputs that may distract the participant from the task at hand.

10. Time, position, and force are recorded during the exploration.

11. After the practice session, the participant is given the option to rest before the actual experiments.

\subsection{Testing Phase}

Participants sit at a table and use the haptic interface to "feel" the unknown object. Half of the participants begin with the one contact point condition and then advance to more contact points according to the ordered list in the Haptic Interfaces section of this paper. The other half of the participants perform the experiments using the interfaces in the reverse order.

Before starting the trial, the contact point corresponding to the index finger is placed on the front (facing the participant) surface of the object halfway along it's line of symmetry. The center of the shape is positioned at $(0,0,0)$, with the $x-y$ plane facing the participant and the $\mathrm{z}$-axis directed at the participant. Thus, in the case of the cone, the center is at its axis of symmetry and half its height. The starting position of the index finger is the point at which the z-axis penetrates the object's surface.

Shapes are randomly introduced to the participant without visual display. Each shape is secured in space and not able to move or rotate. To avoid distractions caused by the surrounding laboratory environment, and to eliminate visual feedback from the exploring hand(s), the participant wears a blindfold. Consequently, the participant "feels" the invisible object in darkness.

Participants are asked to identify the basic shape as fast as possible. Timing starts once the experimenter instructs the participant to begin exploration and ends when the participant indicates that the object has been recognized.

The position of the contact points and the forces exerted by the interface (and conversely the participants because the two forces are in equilibrium) are recorded with respect to time.

Here it is a detailed description of phases during the test: 
1. As in the practice phase, the participant stands with the haptic interface positioned at forearm length directly in front of the torso.

2. The appropriate finger(s) are placed into the thimble of the interface according to either the sequence listed below or the reverse of the sequence. Consequently, half the participants begin with one contact point and continues two contact points while the other half begins with two contact points and continues to one contact point.

3. Throughout the experiment, only the dominant hand is used.

4. A dragging force directs the finger(s) to the object at the beginning of each shape introduction.

5. Four shapes, each sized to fit in a $10 \mathrm{~cm}$ cube, are presented randomly. i. Cone ii. Pyramid iii. Cylinder iv. Triangular extrusion

6. The participants are not be told in advance what shapes will be presented. In the case that the participant is unsure of the name of the shape, a description of the shape will be accepted.

7. As with the practice trial, a spherical bubble of $(9 \mathrm{~cm}$ radius with the same origin as the object) encloses the object to reduce exploration time spent in free space.

8. The participant is told:

"Proceed as you did in the practice session.

Identify the shape as quickly as possible. You will be given a maximum of five minutes to determine the shape. I will ask you to make a guess if we reach the time limit.

Please begin when I say GO."

9. A blindfold is put over the participant's eyes to both block vision of the hand(s) and to eliminate any visual inputs that may distract the participant from the task at hand.

10. Time, position, and force are recorded during the exploration.

11. The dependent variables are as follows:

i. Time to complete the task. Maximum time (three minutes) issued for those asked to make a guess. ii. Percentage correct response. iii. Length of path taken during exploration. iv. Time spent on edges or corners of shape.

\subsection{Data Analysis}

Performance have been analysed based on the following factors:

1. Exploration time

2. Response error

3. Path of exploration

4. Duration exploring particular features of object

5. Force exerted at different stages of exploration, both with respect to time and position

The independent variable for this analysis were the number of contact points. 


\section{Results and discussion}

Figure 4 shows the proportion of the correct answers for the four subjects.

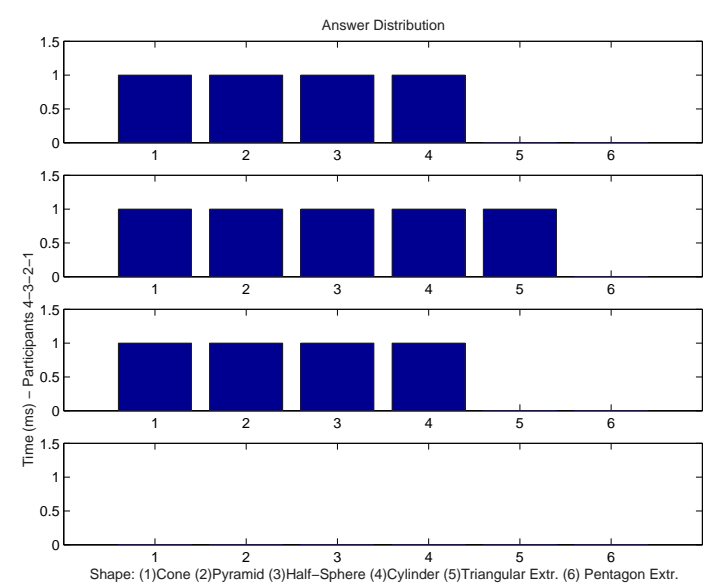

Fig. 4. Proportion of correct answers

From the proportion of correct answers, we see that almost all the simple shapes were correctly identified by all the subjects, while the more complex ones ( 5 and 6 shapes) were difficult to be recognized. This indicate a limit of the haptic interface for the recognition of complex shapes.

The fourth subject was not able in no way to become confident with the interface in all the different conditions. One of the comments reported pointed out the difficulty of identifying the shape, because while the fingertips were constrained on the surface, the hand and finger was able to penetrate within the shape.

Figure 5 reports the summary of exploration time and percentage of contact with the shape for the different fingers. While Figure 5(a) plots the exploration time per subject and per shape necessary to identify the shape, Figure 5(b) plots the percentage of the time spent keeping the contact with the shape for each different finger. From the number of symbols $(o,+, \triangle)$ represented in Figure 5(b), it is possible to reconstruct the associated condition to each experiments (1,2 or 3 contact points). These data are very valuable since allow to judge the quality of the interaction in the different conditions.

From the analysis of Figure 5, it is possible to observe how for each shape, the condition among all the subjects, which required less time for the identification of the shape was the one with one finger, followed by the two and then finally by the three-fingers condition. The only exception is the cone condition \#1, but it we look then carefully at the percent of contact, we discover that almost the contact with the shape was kept through one finger only.

This is confirmed by the direct comparison of the average exploration time for the three conditions, as shown in Figure 6(a). Here the comparison has been limited 


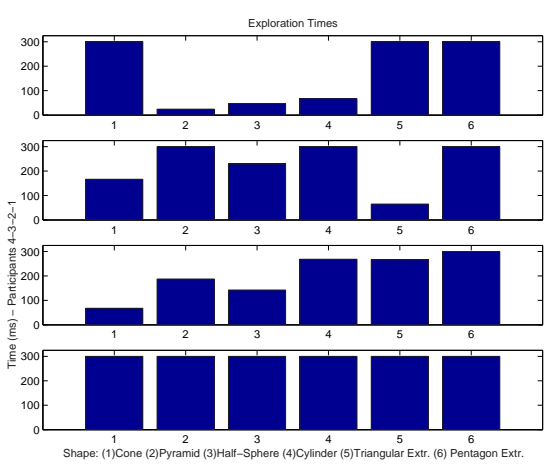

(a) Exploration time

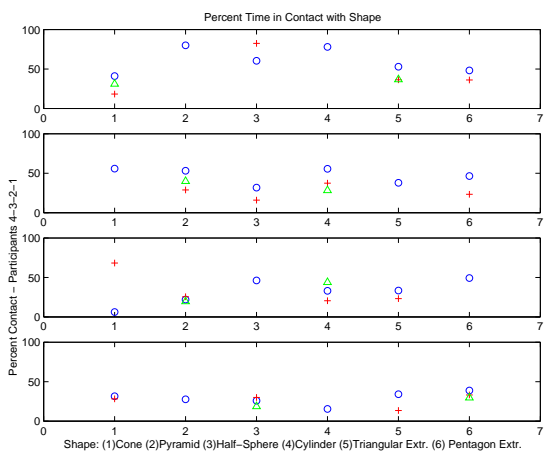

(b) Percentage of contact

Fig. 5. Summary data relative to the three conditions. On the $\mathrm{x}$ axis are reported the shapes associated to each experiment, while on the $y$ axis it is plotted either the performance indicator per participant. In Figure 5(b) each symbol refers to the percentage of contact of each finger (circle $o=$ index, cross $+=$ thumb, triangle $\triangle=$ middle) according to the number of involved fingers.

to the first 3 subjects, since data relative to the $4^{\text {th }}$ subject were not reliable, and to the first four shapes, characterized by simpler geometry and always correctly identified.

The conditions with two fingers does not show up any substantial improvement in the recognition task, and on average the one finger condition results performed better than the two conditions.

We expected that performance, in terms of exploration time and response error, would have improved as more contact points were provided to the participant. Contrary to our expectations, the worse performance came from the 3 fingers condition and the best results came from the one finger condition. These results are coherent with the observations found by (7), with experiments conducted on real object exploration with sheathed fingers. 


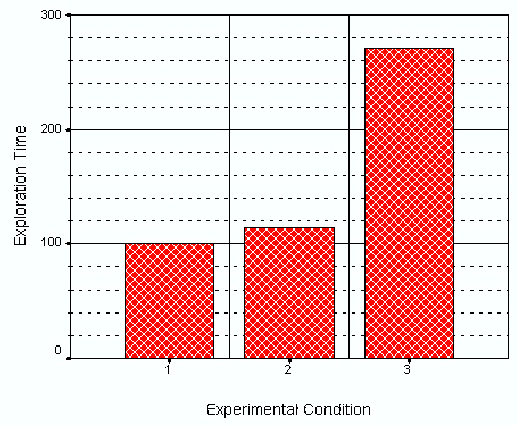

(a) Exploration time

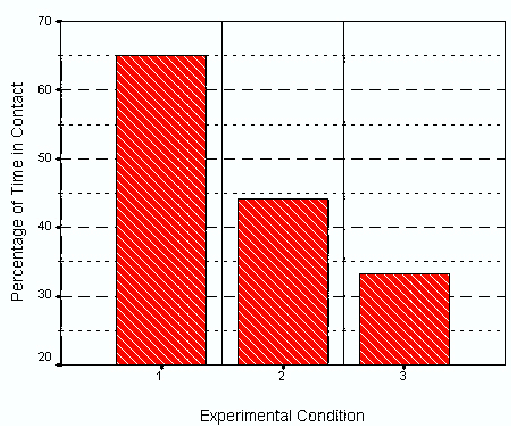

(b) Percentage of contact

Fig. 6. Comparison about the three conditions

The analysis of Figure 6(b), where it is plotted the average percentage time spent in contact for each fingertip versus the different conditions, reveals that having more contact points worsen the ability of subjects to properly follow, keeping the contact, the shape of the virtual objects. This difficulty was also evident during the execution of the experiments, since subjects were systematically loosing the contact with the shape, while exploring the object, the more fingers were involved in the contact. This suggested, with respect to the case of exploration of virtual objects with sheathed fingertips where no difference is noticeable among the different conditions, that some contrasting local force information was provided to each contact point. This can be due to several reasons, mainly by the lack of the tactile feedback on the fingerpad. We however suggest that some specific factors can be pinpointed which account for most of the contrasting information, and they are the following:

- Absence of physical location of the contact on the fingerpad;

- Inhomogenous perception of dynamic properties at each fingertip, due to differences of reflected inertias among different haptic interfaces; 
- Absence of any geometrical information related to the orientation of the contact area.

These points suggested new criteria and guidelines for the design of multipoint haptic interfaces.

\section{Conclusions and Future Examinations}

The present work suggested that current limit of haptic interfaces do not allow to exploit the synergic effect created by the involvement of more fingers during manipulation and exploration of virtual objects. Some criteria were identified that can lead to an improvement of design of multipoint haptic interfaces.

As a future work the incorporation of additional variables such as texture, mass, and stiffness may be interesting, to investigate whether variability in these factors affects shape recognition performance. Does the addition of texture or mass enhance object features and make them easier to recognize or do certain textures act as distractions? Is a more compliant object more recognizable than one that is very stiff? These factors should only be investigated after an understanding of the effects from variables in the study described in this paper is established. 


\section{Bibliography}

[1] S. A. Douglas A. E. Kirkpatrick. Application-based evaluation of haptic interfaces. In Proceedings of the Tenth Symposium on haptic interfaces for virtual environment and teleoperator systems, 2002.

[2] D. Kornbrot C. Colwell, H. Petrie and al. Improving the quality of life for the European citizen: technology for inclusive design and equality, chapter Use of a haptic device by blind and sighted people: perception of virtual textures and objects. IOS Press, Amsterdam, 2003.

[3] C. J. Craig. Attending to two fingers: Two hands are better than one. Perception \& Psycholophysics, 38(6):496-511, 1985.

[4] P. W. Davidson. Haptic judgments of curvature by blind and sighted humans. Journal of Experimental Psychology, 93(1):43-55, 1972.

[5] A. Frisoli, F. Simoncini, and M. Bergamasco. Mechanical design of a haptic interface for the hand. In to appear in 2002 ASME International DETC-27th Biennial Mechanisms and Robotics Conference, 2002.

[6] G. Jansson. Effects of number of fingers involved in exploration on haptic identification of objects. excerpt from pure-form: The museum of pure form; haptic exploration for perception of the shape of virtual objects. Technical report, EU-PURE FORM, 2000.

[7] G. Jansson. Pure- form 4th period 6-monthly report. Technical report, Upssala University, 2004.

[8] G. Jansson and L. Monaci. Touch, Blindness and Neuroscience, chapter Haptic identification of objects with different numbers of fingers. UNED press, Madrid, 2003.

[9] R. L. Klatzky and al. Vision and action: The control of grasping., chapter Procedures for Haptics Object Exploration vs. Manipulation, pages 110-127. 1990.

[10] R. L. Klatzky and al. Haptic identification of objects and their depictions. Perception \& Psychophysics, 54(2):170-178, 1993.

[11] R. L. Klatzky and S. J. Lederman. Tactile roughness perception with a rigid link interposed between skin and surface. Perception \& Psychophysics, 61(6):591607, 1999.

[12] R. L. Klatzky, S. J. Lederman, and V. A. Metzger. Identifying objects by touch: An “expert system”. Perception \& Psychophysics, 37(4):299-302, 1985. 